\title{
Investigation of the Klinkenberg effect in a micro/nanoporous medium by direct simulation Monte Carlo method
}

\author{
Guang Yang ${ }^{1,2, *}$ and Bernhard Weigand ${ }^{2}$ \\ ${ }^{1}$ Institute of Refrigeration and Cryogenics, Shanghai Jiao Tong University, 200240 Shanghai, China \\ ${ }^{2}$ Institut für Thermodynamik der Luft- und Raumfahrt, Universität Stuttgart, 70569 Stuttgart, Germany
}

(Received 28 November 2017; published 20 April 2018)

\begin{abstract}
The pressure-driven gas transport characteristics through a porous medium consisting of arrays of discrete elements is investigated by using the direct simulation Monte Carlo (DSMC) method. Different porous structures are considered, accounting for both two- and three-dimensional arrangements of basic microscale and nanoscale elements. The pore scale flow patterns in the porous medium are obtained, and the Knudsen diffusion in the pores is studied in detail for slip and transition flow regimes. A new effective pore size of the porous medium is defined, which is a function of the porosity, the tortuosity, the contraction factor, and the intrinsic permeability of the porous medium. It is found that the Klinkenberg effect in different porous structures can be fully described by the Knudsen number characterized by the effective pore size. The accuracies of some widely used Klinkenberg correlations are evaluated by the present DSMC results. It is also found that the available correlations for apparent permeability, most of which are derived from simple pipe or channel flows, can still be applicative for more complex porous media flows, by using the effective pore size defined in this study.
\end{abstract}

DOI: 10.1103/PhysRevFluids.3.044201

\section{INTRODUCTION}

Fluid flow in porous media with micro- and nanopores has attracted numerous investigations due to its great importance in a wide range of application fields [1,2], including $\mathrm{CO}_{2}$ sequestration, unconventional gas production, hydrogen storage, etc. The emerging MEMS technology, where complex networks of microchannels are often used, also brings great demands for the study of micro- and nanoscale gas transport mechanisms [3]. For gas flow in such small domains, the collisions between gas molecules and solid walls are significant, and the velocity profile behaves differently compared to that predicted by conventional macroscopic theory. This phenomenon is also called Knudsen diffusion and is quantified by the Knudsen number, which is defined as the ratio of the mean free path length $(\lambda)$ of gas molecules to one characteristic dimension of the flow $\left(L_{\mathrm{ch}}\right.$, can be chosen as diameter, pore size, etc.), i.e. $\mathrm{Kn}=\lambda / L_{\mathrm{ch}}$. Based on the Knudsen number, the gas flow can be empirically classified to four regimes: continuum flow $(\mathrm{Kn} \leqslant 0.001)$, slip flow $(0.001<\mathrm{Kn} \leqslant 0.1)$, transition flow $(0.1<\mathrm{Kn} \leqslant 10)$, and free-molecular flow $(\mathrm{Kn}>10)$. For fluid flow in continuum and slip flow regions, the Navier-Stokes equations are applicable, with no-slip boundary condition or with appropriate velocity-slip/temperature-jump boundary conditions. However, for fluid flow in

\footnotetext{
*y_g@sjtu.edu.cn, guang.yang@itlr.uni-stuttgart.de
}

Published by the American Physical Society under the terms of the Creative Commons Attribution 4.0 International license. Further distribution of this work must maintain attribution to the author(s) and the published article's title, journal citation, and DOI. 
the transition and free molecular flow regions, the continuum hypothesis is no longer applicable, and the Boltzmann equations should be adopted to describe the flows.

A particle-based method, direct simulation Monte Carlo [4] (DSMC) is a promising method for flows in the transition and free molecular flow regions, and it has been validated against experimental data in a wide range of Knudsen numbers [5,6]. DSMC converges to the Boltzmann equations [7] based on direct statistical simulations of molecular processes described by the kinetic gas theory. As DSMC replaces the deterministic motion by a stochastic approximation for the collision process, it is more efficient than the molecular dynamic (MD) method for certain problems. On the other hand, due to its high accuracy, the results simulated by DSMC have also been used frequently as benchmarks to validate other numerical methods, such as the Lattice Boltzmann method [8].

From the macroscopic view, the rarefaction effect caused by the Knudsen diffusion in the pore scale leads to the increase of the gas permeability (apparent permeability) from the intrinsic permeability of the porous medium, which is known as the Klinkenberg effect. Klinkenberg [9] derived the expression for the apparent permeability as $K_{a}=K_{\infty}(1+b / P)$, where $b$ is the correction factor. This correlation has been a consistent basis for the developments of several new correlations [10]. To predict the Klinkenberg effect, it is significant to accurately obtain the pore scale flow patterns in the porous medium and then use an upscaling method. A few examples can be found in the literature where the DSMC method is used to solve gas transport problems in porous media. Stern et al. [11] performed microscale simulations of spacecraft thermal protection system (TPS) materials. They showed that their simulations by DSMC were feasible at relevant scales and conditions. Kawagoe et al. [12] simulated pressure-driven gas flow through randomly arranged solid spherical particles with the porosity ranging from 0.3 to 0.5 by DSMC. It was confirmed that Darcy's law was valid even in the case of porous media with micro- and nanoscale pores. Christou and Dadzie [13] performed a DSMC study for Berea sandstone for a pressure driven flow of $\mathrm{CH}_{4}$ at different Knudsen numbers, and $\mathrm{Kn}$ was found to play an important role for the velocity profiles. Borner et al. [14] computed the permeability of several fibrous substrates to high-temperature gases. The actual porous geometry of the materials was digitized using $X$-ray microtomography and the range of the porosity was 0.8 to 0.9 .

The objective of this work is to provide a deeper understanding of the gas transport characteristics through a micro- and nanoporous medium by DSMC. On the basis of pore scale flow patterns, the apparent permeability will be further investigated in detail. Another important aim is to assess the accuracies of different available Klinkenberg correlations, most of which were proposed based on simple geometries of channels or tubes, and to find a possible way to link them with a more complex porous media flow. The rest of this paper is organized as follows: in Sec. II, the physical model of interest is introduced; in Sec. III, the DSMC method is described in detail, some numerical parameters are provided, and the effective pore size of a porous medium is defined; in Sec. IV, the results are firstly presented for a porous medium with 2D array of square elements, the local flow patterns under various Knudsen numbers and porosities are obtained, the apparent permeabilities are calculated, and the available Klinkenberg correlations are evaluated for different porous structures and for 3D arrangements. The paper closes with some key conclusions in Sec. V.

\section{PHYSICAL MODEL}

Figure 1 illustrate an example of a two-dimensional idealized porous medium along with the relevant dimensions considered in the present study. A large number of solid elements are arranged periodically, which form a porous medium. The distance between neighboring elements is $H$. The geometry is in the atmosphere of nitrogen gas with a temperature of $T_{\mathrm{in}}$. The porosity is defined as the fraction of pore space $\Omega_{f}$ in the porous medium of total volume $\Omega$. For elements with square cross-sections, as shown in Fig. 1, the porosity of the porous medium is calculated as

$$
\varepsilon=\frac{\Omega_{f}}{\Omega}=1-\frac{D^{2}}{H^{2}} .
$$




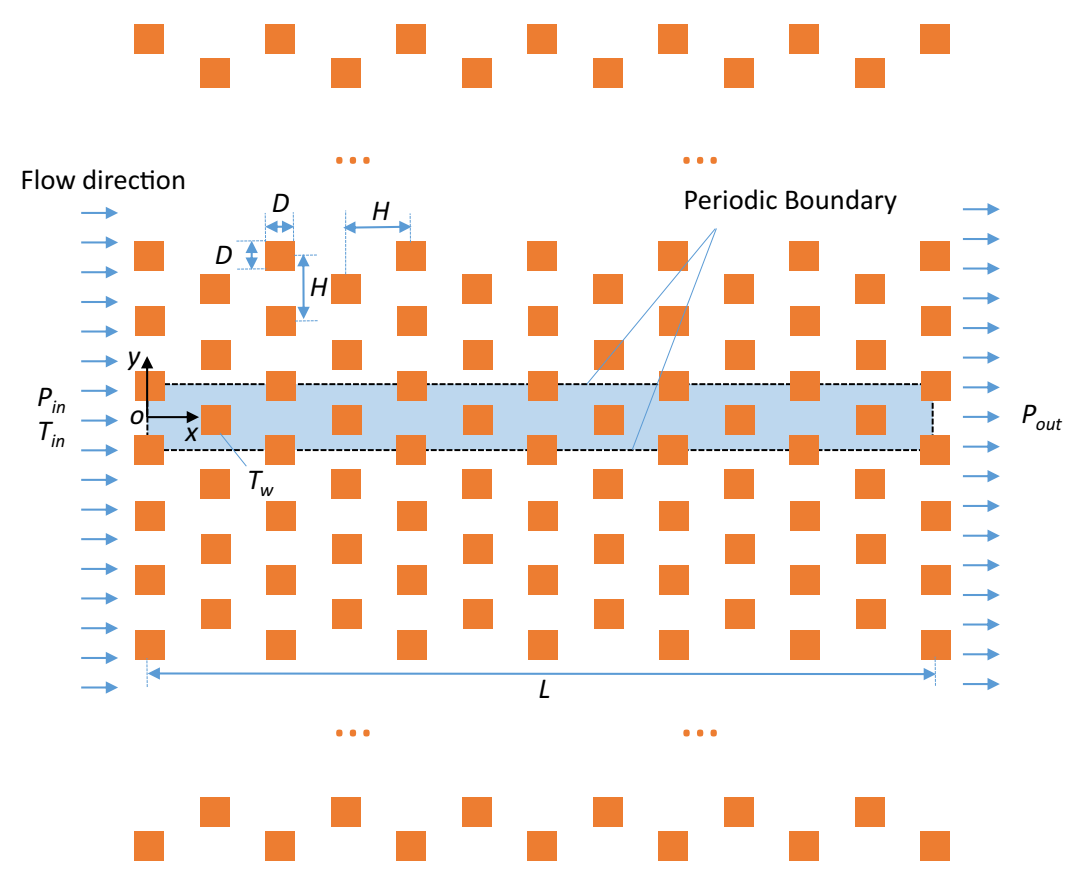

FIG. 1. Schematics of flow past arrays of two-dimensional square elements.

The geometry is considered infinite in the $Y$ direction but of finite length in the $X$ direction. Therefore, a computation domain of $H \times L$ is adopted in this study (see Fig. 1). Periodic boundary conditions are used on the upper and lower boundaries. The pressures on the left and right sides of the porous medium are $P_{\text {in }}$ and $P_{\text {out }}$, respectively $\left(P_{\text {in }}>P_{\text {out }}\right)$. Even though a periodic boundary could also be used in the $X$ direction for the gas inlet and outlet, which would reduce the computation size, the usage of pressure boundaries are more in accordant with practical situations as flows in nanoand microchannels are typically driven by large pressure gradients [15].

The effect of the length of the computational domain $L$ on the permeability of the porous medium is also checked, to choose a proper computation domain (detailed results are shown in Appendix A). $L=12 \mathrm{H}$ has been proven to be sufficient to represent a porous medium. The temperatures of the inlet gas $\left(T_{\mathrm{in}}\right)$ and at the surfaces of the square elements $\left(T_{w}\right)$ are both equal to $300 \mathrm{~K}$ in this study.

\section{METHOD}

\section{A. DSMC method}

The investigation is performed by using the direct simulation Monte Carlo (DSMC), which is a particle-based method performed by modeling independent simulated particles [4]. The DSMC code used in the present work has been implemented in OpenFOAM, an open-source $\mathrm{C}++$ tool box for computational fluid dynamics $[6,16]$. The computation domain is divided into a large number of grid cells, and the cell length is carefully chosen such that it is a fraction $(1 / 3$, in this study) of the smallest mean free path. The time step $(\Delta t)$ for the simulations is also chosen as a fraction $(1 / 5$, in this study) of the mean collision time in DSMC [17]. To reduce statistical scatter and to ensure that collisions are performed accurately, a minimum of 10 particles per computational cell are used. As it can be inferred from the study of Sun et al. [18], the maximum relative difference of velocities between the cases using 10 particles per cell and 20 particles per cell is less than $1 \%$. Diffuse wall reflection boundaries are adopted for the solid surfaces of the square elements. The collision pairs are selected 
using the no-time-counter (NTC) method, and the collisions between particles are simulated using variable hard sphere (VHS) collision model and Larsen-Borgnakke internal energy redistribution model [4].

\section{B. Inlet and outlet boundary conditions}

Gas flows in a micro- and nanoporous medium are typically of low velocities and driven by a pressure gradient. It is likely that particles will have thermal velocities orders of magnitude larger than the stream velocity. This may make it likely that fluids do not always flow in the streaming direction. Due to experimental limitations, the microscale velocity profiles are usually not known at the boundaries. Therefore, the pressure boundary condition is adopted in the present simulation, where the inlet pressure, inlet temperature, and outlet pressure are set up before calculation. The number flux of the particles entering the computational domain can be described from the equilibrium Maxwell-Boltzmann distribution:

$$
\begin{gathered}
\dot{n}=\frac{n V_{\mathrm{mp}}}{2 \sqrt{\pi}}\left[\exp \left(-s^{2} \cos \theta^{2}\right)+\sqrt{\pi} s \cos \theta\{1+\operatorname{erf}(s \cos \theta)\}\right], \\
s=\frac{V}{V_{\mathrm{mp}}}, \\
V_{\mathrm{mp}}=\sqrt{\frac{2 k_{B} T}{m}},
\end{gathered}
$$

where "erf" represents the error function. $\theta$ is the angle between the velocity vector and normal to the boundary element. $V$ is the local stream velocity, $V_{\mathrm{mp}}$ is the local most probable thermal velocity, $T$ is the macroscopic temperature at the boundary, and $k_{B}$ is the Boltzmann constant. The implicit velocity treatment by Wang and $\mathrm{Li} \mathrm{[19]} \mathrm{is} \mathrm{used} \mathrm{at} \mathrm{the} \mathrm{inlet,} \mathrm{where} \mathrm{statistical} \mathrm{macroscopic} \mathrm{velocity}$ is obtained from particles in the cells near the inlet boundary. The outlet boundary is treated by the method proposed by Liou and Fang [20].

\section{Solution procedure}

The DSMC is performed either in two- or three-dimensional in this study, depending on the porous structures. A typical procedure of the DSMC method consists of indexing particles into cells, tracking the movement of particles, computing collisions, and calculating post-collision properties. These steps are repeated to increase the sample size until the statistical errors are small enough. The macroscopic flow characteristics are obtained by statistically sampling and averaging the molecular properties in each cell. For example, the density, velocity, and overall temperature can be calculated as

$$
\begin{gathered}
\rho=n \bar{m}=n \frac{1}{N} \sum_{i=1}^{N} m_{i}, \\
V=\frac{1}{N} \sum_{i=1}^{N} V_{i}, \\
T=\frac{3 T_{r}+\zeta T_{\text {rot }}}{3+\zeta},
\end{gathered}
$$

where $n, m, N, T_{r}, T_{\text {rot }}$, and $\zeta$ are number density, molecular mass, number of particles in the cell during the specific time, translational temperature, rotational temperature, and the number of rotational degrees of freedom, respectively. 
At the initial time, the simulated particles are uniformly distributed statistically in the cells in the computation domain, at the state of $\left(P_{\text {in }}+P_{\text {out }}\right) / 2$ and $T_{\text {in }}$. The number of DSMC particles and the average linear kinetic energy are monitored in the simulations. After the flow reach steady states (The solution was considered to be steady when the two monitored parameters do not change over time [16]), time averaging of the instantaneous microscopic properties is performed to build up the macroscopic fields.

The magnitude of velocity fluctuation of the flow velocity can be estimated to be $\left.\left(1 / \sqrt{N_{\text {total }}}\right) \sqrt{k_{B} T / m}\right)$, where $N_{\text {total }}\left(=\sum_{i=1}^{N_{\text {samp }}} N^{i}, N_{\text {samp }}=t_{\text {samp }} / \Delta t\right)$ is the total number of sampled particles. Therefore, the uncertainty of the velocity can be reduced by increasing number of sampled particles, i.e., increasing sampling time $\left(t_{\text {samp }}\right)$. The smaller the average velocity in the computation domain, the longer the sampling time is needed. In this study, the time for averaging in each case is determined to make the statistic uncertainty of average velocity less than $0.1 \%$.

All cases in this study are simulated on a cluster with dual processor Intel(R) Xeon(R) CPU E5-2690 v4 at $2.60 \mathrm{GHz}$. In total 28 nodes are used for each case. The computational time has been found to greatly increase as the Knudsen number decreases. For example, the computation times is about $350 \mathrm{~h}$ for the case of $\mathrm{Kn}_{D}=0.0455$ and about $15 \mathrm{~h}$ for $\mathrm{Kn}_{D}=0.445$ at $\varepsilon=0.75$.

\section{Knudsen number and effective pore size}

The extent of gas rarefaction is characterized by the Knudsen number Kn,

$$
\mathrm{Kn}=\frac{\lambda}{L_{\mathrm{ch}}},
$$

where $\lambda$ denotes the mean free path of gas molecules, and can be expressed as

$$
\lambda=\frac{k_{B} T}{\sqrt{2} \pi d^{2} P},
$$

where $k_{B}$ is the Boltzmann constant, and $d$ the molecule diameter. According to the VHS model [4], the mean free path for a real gas accounting for a temperature coefficient $\omega$ can be expressed as

$$
\lambda=\frac{2(5-2 \omega)(7-2 \omega)}{15}\left(\frac{m}{2 \pi k T}\right)^{\frac{1}{2}}\left(\frac{\mu}{\rho}\right)
$$

$L_{\mathrm{ch}}$ in Eq. (8) is a characteristic length of the flow. In previous studies concerning flow through a porous medium, various characteristic lengths have been used [21-24]. In this study, we use both the size of the elements $(D)$, as many studies in literatures did, and also the pore size $\left(D_{p}\right)$ to define the Knudsen numbers:

$$
\begin{aligned}
\mathrm{Kn}_{D} & =\frac{\lambda}{D}, \\
\mathrm{Kn}_{p} & =\frac{\lambda}{D_{p}} .
\end{aligned}
$$

Due to the great variety and complex connections of the pore structure, the actual pore size that affect the permeability of a porous medium cannot be obtained straightforward by measuring the hydraulic diameter/radius. Therefore, an effective pore size should be defined.

By analogy with electrical conductance, the intrinsic permeability of a porous medium can be fully described by the combined effects of the porosity, the characteristic length, the tortuosity, and the constriction factor [25].

$$
K_{\infty}=\frac{\tau^{2} L_{\mathrm{ch}}^{2} \varepsilon}{8 C}
$$


where $K_{\infty}$ is the intrinsic permeability of the porous medium. $\tau$ in Eq. (13) is the tortuosity of the porous structure [26], which is defined as the length of the porous medium $(L)$ divided by the length of the streamline $\left(L_{s}\right)$ :

$$
\tau=\frac{L}{L_{s}} .
$$

Based on this definition, $\tau$ is always less than or equal to 1 . A smaller tortuosity indicates the fluid needs to travel longer in the porous medium for a fixed L. C in Eq. (13) is the constriction factor of the porous structure, which represents the effect of the variation in cross-sectional area [27]:

$$
C=\frac{1}{L^{2}} \int_{0}^{L} A(x) d x \int_{0}^{L} \frac{1}{A(x)} d x,
$$

where $A(x)$ is the cross-sectional area of the flow path at point $x$. It can be inferred from Eqs. (1), (14), and (15), that $C, \tau$, and $\varepsilon$ depend only on pore structures and the flow direction.

As is well known, the intrinsic permeability of a porous medium is also only a function of material, regardless of the flow field. Therefore, the effective pore size $\left(D_{p}\right)$ of a porous medium can be defined as

$$
D_{p}=\sqrt{\frac{8 K_{\infty} C}{\tau^{2} \varepsilon}} .
$$

As can be inferred from above, $D_{p}$ is also a property of the porous structures.

\section{E. Data reduction}

As the mean free path of gases $\lambda$ varies with gas pressure [see Eq. (9)], local Knudsen numbers change significantly from inlet to outlet of the geometry for a pressure driven flow. The average Knudsen numbers $\left(\overline{\mathrm{Kn}_{D}}\right.$ and $\left.\overline{\mathrm{Kn}_{p}}\right)$ are also defined, by replacing $\lambda$ in Eqs. (11) and (12) with the average mean free path $\bar{\lambda} \cdot \bar{\lambda}$ is calculated based on the properties for the average pressure $\bar{P}$ in the porous medium. Though the variation of $P$ is not perfectly linear in the geometry (Appendix B), $\bar{P}$ is simply calculated as $\bar{P}=\left(P_{\text {in }}+P_{\text {out }}\right) / 2$, as it is more feasible in practical applications [12,28].

Based on Darcy's equation, the apparent permeability $\left(K_{a}\right)$ of the porous medium for a pressuredriven isothermal flow of ideal gas can be calculated as [29]

$$
K_{a}=\frac{2 \mu L P_{\text {out }} \overline{V_{x, \text { out }}}}{P_{\text {in }}^{2}-P_{\text {out }}^{2}}
$$

where $\overline{V_{x, \text { out }}}$ is the macroscopic average velocity at the outlet of the porous medium:

$$
\overline{V_{x, \text { out }}}=\frac{\int_{-H / 2}^{H / 2} V_{x} d Y}{H} .
$$

\section{RESULTS AND DISCUSSION}

\section{A. Local flow patterns}

The distributions of the velocity magnitude, along with the streamlines, in the two-dimensional square element array (pores of the porous medium) for different element sizes $D=1 \mu \mathrm{m}, 400 \mathrm{~nm}$, $100 \mathrm{~nm}$, and $20 \mathrm{~nm}$ are presented in Figs. 2(a)-2(d), under the same boundary condition of $P_{\text {in }}=0.25 \mathrm{MPa}$ and $P_{\text {out }}=0.05 \mathrm{MPa}$ and at a fixed porosity of $\varepsilon=0.75$. The corresponding average Knudsen numbers are $\overline{\mathrm{Kn}_{D}}=0.0445,0.111,0.445$, and 2.23 , respectively. As is driven by the pressure difference between the inlet and outlet, the nitrogen flows from left to right. For each case, the maximum velocity distributes near the outlet. This is because the mass flow rate in each cross section is constant, but as a lower local pressure leads to a lower density of the fluid, the volume flow rate increases along the flow direction. It can also be inferred from the legends of Figs. 2(a)-2(d), 


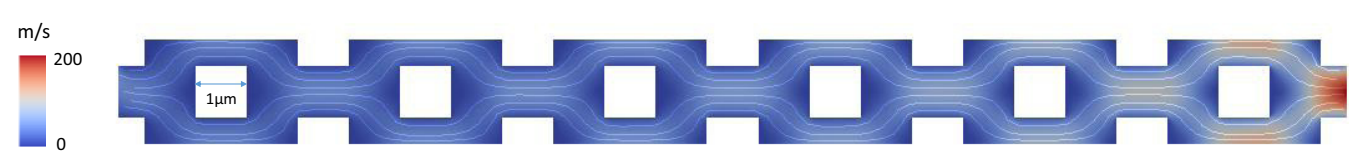

(a)

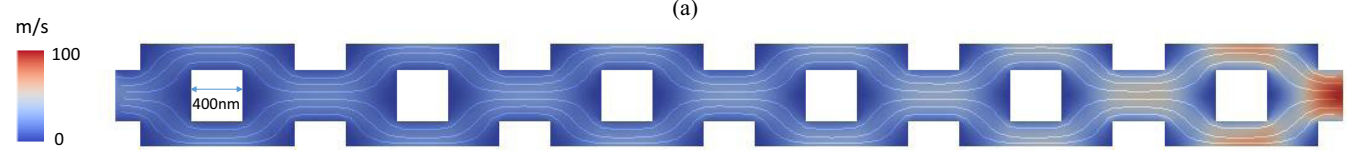

(b)

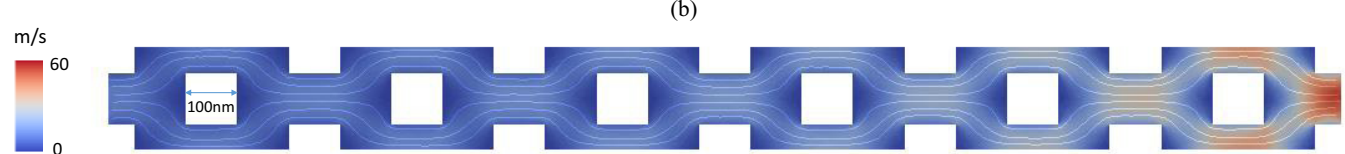

(c)
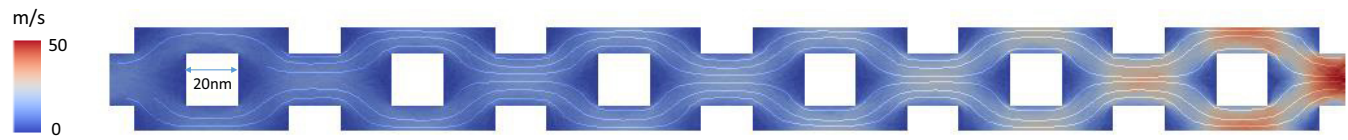

(d)

FIG. 2. Velocity distribution and streamlines for $D=$ (a) $1 \mu \mathrm{m}$, (b) $400 \mathrm{~nm}$, (c) $100 \mathrm{~nm}$, and (d) $20 \mathrm{~nm}$, $\varepsilon=0.75$.

that the flow rate through the porous medium decreases with decreasing pore sizes, under a given pressure difference.

To study the velocity profiles in more detail, the distributions of the velocity in axial direction $\left(V_{x}\right)$ at different locations $(X=2 H, 4 H, 6 H, 8 H$, and $10 H)$ for $\varepsilon=0.75, D=400 \mathrm{~nm}, P_{\text {in }}=$ $0.25 \mathrm{MPa}$, and $P_{\text {out }}=0.05 \mathrm{MPa}$ [Fig. 2(b)] are presented in Fig. 3(a). Because of the Knudsen diffusion, the gas velocities at the solid walls are always nonzero. With increasing axial distances, the "slip" velocity at the walls increases, due to the decrease of local pressures (increasing local Knudsen number). The centerline velocity is also found to increase accordingly. The axial velocity $\left(V_{x}\right)$ distribution at $X=6 H$ for different element sizes of $D=1 \mu \mathrm{m}, 400 \mathrm{~nm}, 100 \mathrm{~nm}$, and $20 \mathrm{~nm}$ and $\varepsilon=0.75$ are presented in Fig. 3(b). The difference in the "slip" velocity at the walls for the different cases is small, but the velocity at the centerline is strongly increased with increasing $D$. Therefore, the variation of the velocity along $Y$ is less pronounced for smaller $D$.

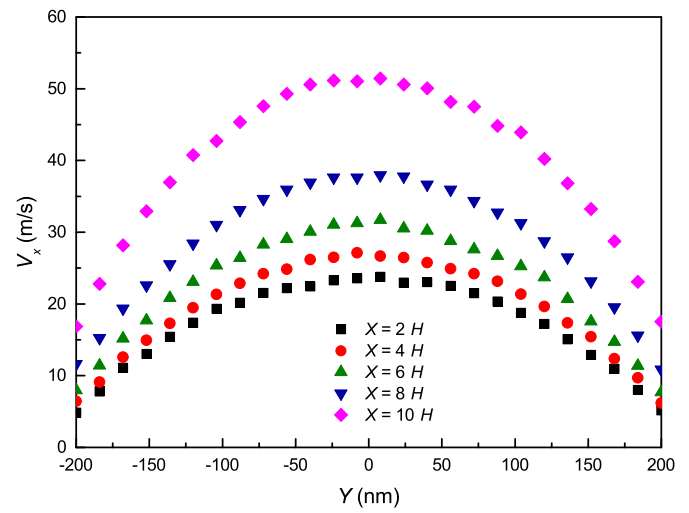

(a)

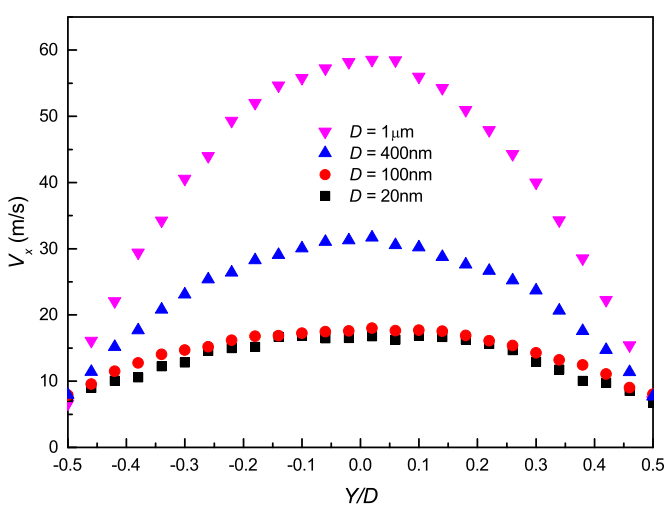

(b)

FIG. 3. Distribution of $V_{x}$ along $Y$ for different (a) locations and (b) element sizes. 


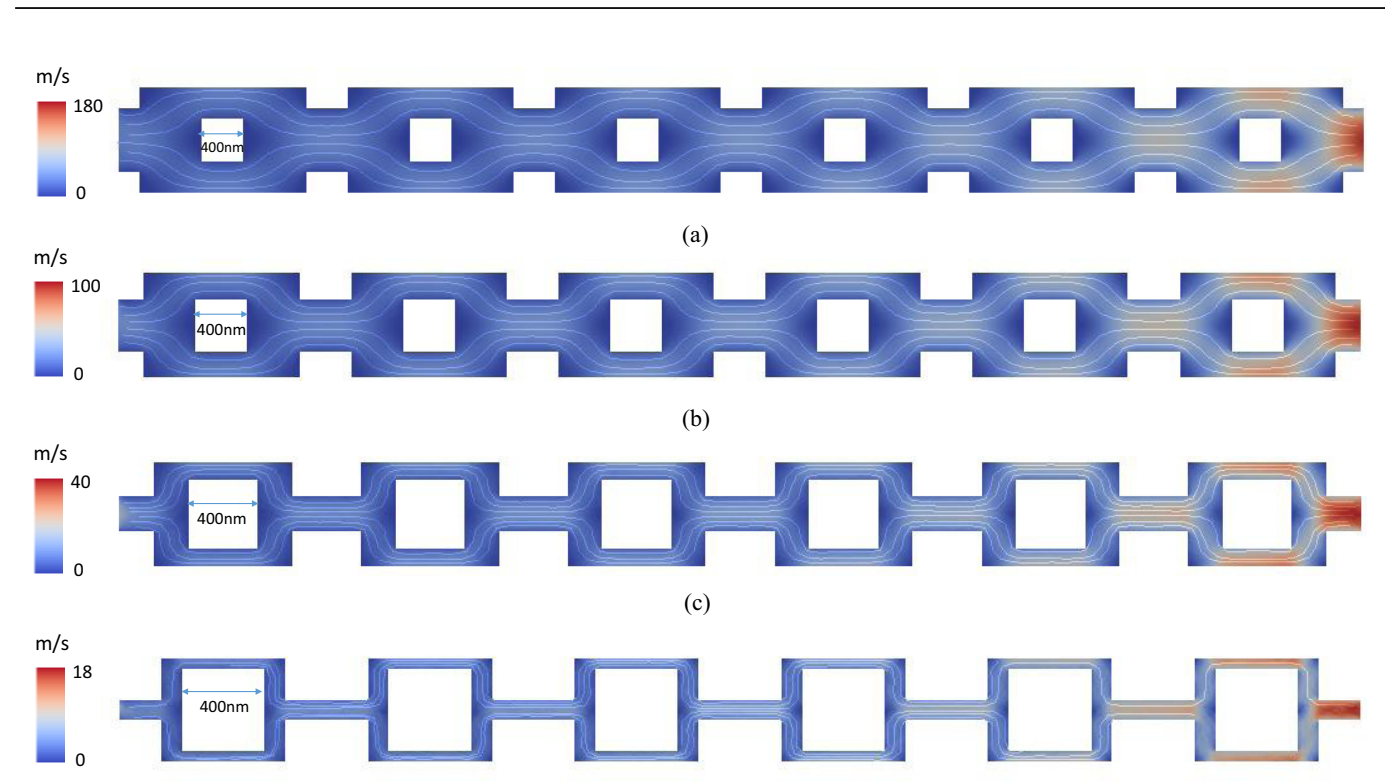

(d)

FIG. 4. Velocity distribution and streamlines for $\varepsilon=$ (a) 0.84 , (b) 0.75 , (c) 0.556 , and (d) $0.36, D=400 \mathrm{~nm}$.

The velocity profiles, along with the streamlines, in the porous medium for different porosities of $\varepsilon=0.84,0.75,0.56$, and 0.36 , at $D=400 \mathrm{~nm}$, and for $P_{\text {in }}=0.25 \mathrm{MPa}$ and $P_{\text {out }}=0.05 \mathrm{MPa}$ are presented in Figs. 4(a)-4(d). The flow rate is found to decrease drastically with decreasing porosity for a given $D$. This is because the size of the pores, where gas flows, is decreased as the decrease of porosity. It can also be found from Figs. 2 and 4 that the statistical noises in the velocity fields are quite small for all the cases, even when the velocity is low.

\section{B. Apparent permeability for various porosities}

Due to the velocity slip at the walls for $\mathrm{Kn}>0.001$, the permeability of the porous medium for gas flow (apparent permeability) is always higher than the intrinsic permeability, which is called as Klinkenberg effect. Prior to the study of the Klinkenberg effect, the intrinsic permeabilities for the geometry in Fig. 1 for different porosities were calculated. Numerical simulations were performed using water as flow medium and using a conventional finite-volume method based on the continuity and Navier-Stokes equations. The detailed numerical procedure can be found in other works [30]. The results of the dimensionless intrinsic permeabilities $K_{\infty} / D^{2}$, determined from the linear form of Darcy's law, are presented in Fig. 5. The results are also validated by the studies of Aerov and Tojec [31], Drummond and Tahir [32], Gebart [33] and Yazdchi et al. [34], and show very good agreement.

Based on the DSMC results for different Knudsen numbers, the apparent permeability for various porosities are calculated by Eq. (17), and the ratio of $K_{a}$ and $K_{\infty}$ are plotted for different $\mathrm{Kn}_{D}$ in Fig. 6. For a fixed porosity, $K_{a} / K_{\infty}$ increases monotonically with increasing $\mathrm{Kn}_{D}$, due to the increased Knudsen diffusion effect, as was previously shown in Fig. 2. $K_{a} / K_{\infty}$ is also found to decrease with decreasing porosity, if $\mathrm{Kn}_{D}$ is kept constant. This is because even though $\mathrm{Kn}_{D}$ is fixed, decreasing porosity decreases the pore size, thus the collisions between gas molecules and solid walls are enhanced. Therefore, the Knudsen number characterized by the element size $\left(\mathrm{Kn}_{D}\right)$ is not able to reflect the "effective" Knudsen diffusion in the porous medium, if various porosities are considered. A similar result was also presented in the work of Kalarakis et al. [41] by using the characteristic length of $4 \varepsilon / S_{v}$ in the Knudsen number, where $S_{v}$ is the internal surface area per unit volume.

The variation of $K_{a} / K_{\infty}$ with $\mathrm{Kn}_{p}$, for $\varepsilon=0.36,0.556,0.75$, and 0.84 , is shown in Fig. 7 . As the effective pore size in $\mathrm{Kn}_{p}$ concerns all the properties of the porous structure Eq. (16), $K_{a} / K_{\infty}$ for 


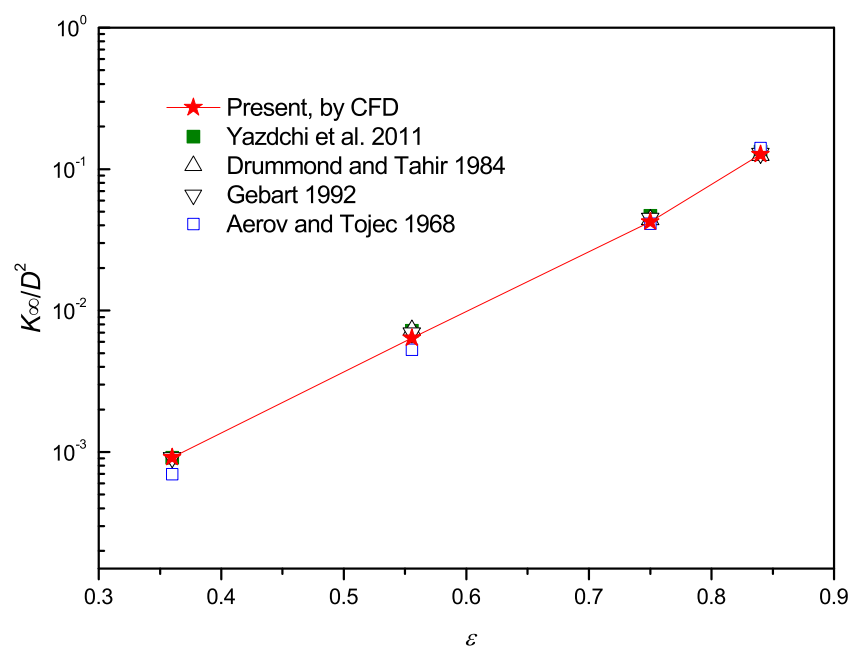

FIG. 5. Dimensionless intrinsic permeability for various porosities [31-34].

various porosities lie almost on one curve with $\mathrm{Kn}_{p}$, as shown in Fig. 7. Furthermore, some widely used Klinkenberg correlations or models, as listed in Table I, are also presented in Fig. 7 (lines 1-7) to compare with present DSMC results. The variation trends of $K_{a} / K_{\infty}$ with $\mathrm{Kn}_{p}$ by the available correlations are found similar with present data for $0.01<\mathrm{Kn}_{p}<10$. Outstandingly, the predicted values by Knudsen [35], Sakhaee and Bryant [36], Civian [10], and Beskok and Karniadakis [37] are very close to the present data, with average relative deviations of $5.37 \%, 6.75 \%, 9.00 \%$, and $9.42 \%$, respectively. Please note that the line 8 in Fig. 7 is an analytical equation, which has been developed only under the assumption of slip flow regime. It proves that the slip assumption at the solid walls becomes invalid if $\mathrm{Kn}_{p}>0.1$.

It should be noted that most of the Klinkenberg correlations listed in Table I are derived from simple pipe or channel flows. In a recent study of Wu et al. [24], it was pointed out that all the currently widely used empirical solutions, derived from straight cylindrical tube, should be reformulated for a porous medium. However, the results in Fig. 7 prove that these correlations are still applicative for flows

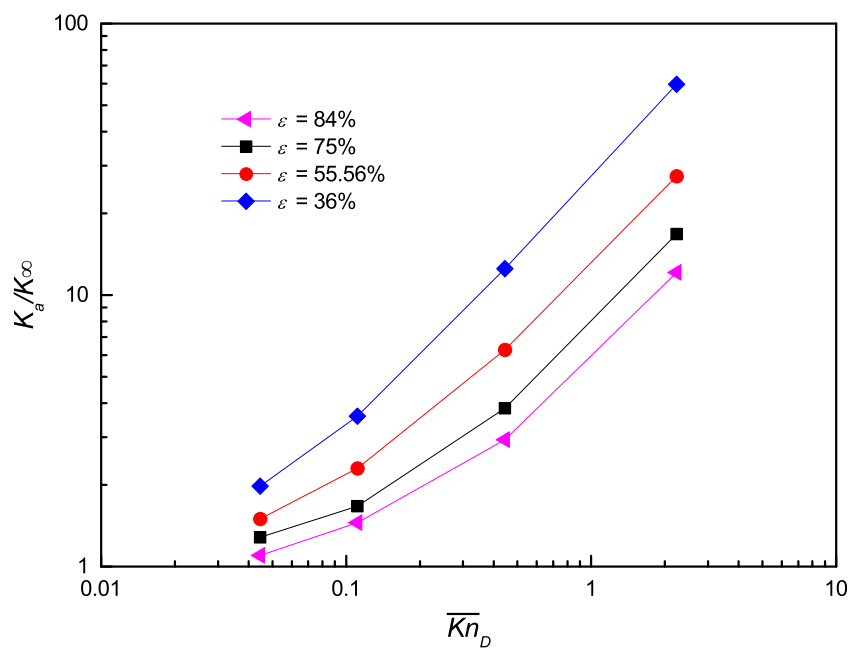

FIG. 6. Variation of $K_{a} / K_{\infty}$ with $\mathrm{Kn}_{D}$ for various porosities. 


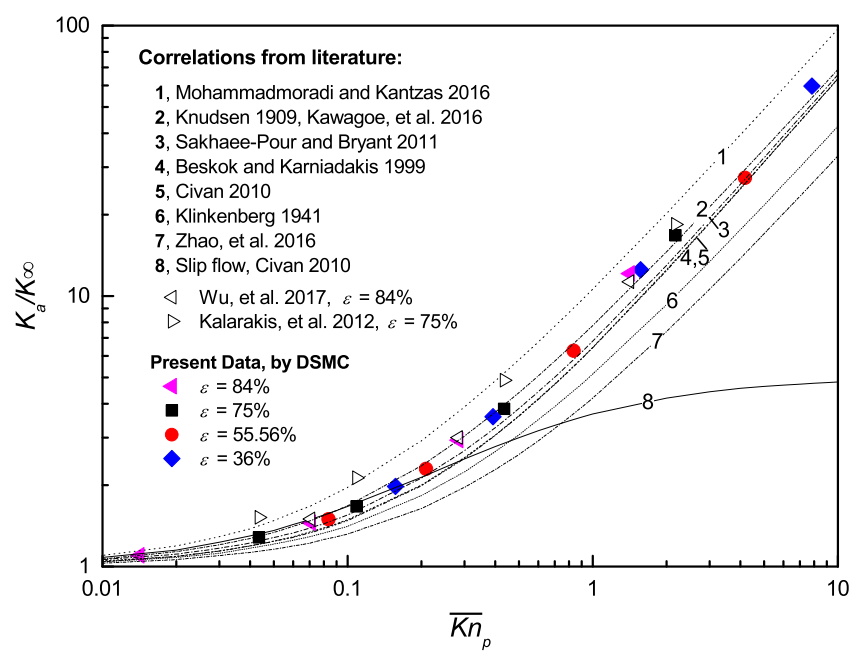

FIG. 7. Variation of $K_{a} / K_{\infty}$ with $\mathrm{Kn}_{p}$ for various porosities [8-10,12,23,24,35-37,41].

in more complex geometries, provided that an appropriate Knudsen number is used. Furthermore, some data for the porosity of $\varepsilon=0.84$ by Wu et al. [24], which were originally plotted with another definition of Knudsen number, are converted to the present $\mathrm{Kn}_{p}$. It can be seen in Fig. 7 that their result can also fit well with present data and with these correlations.

There are also available correlations considering the Klinkenberg effect obtained from more complex natural porous media (such as sandstones, reservoirs), which are provided in the form of

$$
K_{a} / K_{\infty}=1+\frac{b}{P}
$$

Jones [39] experimentally found $b \propto K_{\infty}^{-0.36}$; Bravo [40] considered $b$ as a function of $K_{\infty}$ and the Knudsen diffusivity coefficient, and the latter could also be correlated as a function of $K_{\infty}$. However, the effect of pore-scale geometry features was not included in these correlations. Therefore, these correlations are accurate only for porous media of specific microstructures. Kalarakis et al. [41] improved the expression of $b$ on the basis of Bravo [40] by including a numerical factor $a$ for the length-to-width ratio of the pore. However, that study only gave empirical values of $a=3.4$ and 4 for the porous structures used in their study with $\varepsilon=0.7$ and 0.8 , the expressions to fully describe the geometry features were unavailable. By using an estimated value of $a=3.5$, the expression of

TABLE I. Correlations of $K_{a} / K_{\infty}$ with Kn from literature.

\begin{tabular}{lcc}
\hline \hline Ref. & $f(\mathrm{Kn})$ & Remark \\
\hline Knudsen, 1909 [35], Kawagoe et al., 2016 [12] & $1+\frac{64}{3 \pi} \frac{1+c_{1}^{K} p}{1+c_{2}^{K} p} \mathrm{Kn}$ & $c_{1}^{K} p=\sqrt{\frac{\pi}{2}} \frac{2}{\mathrm{Kn}} ; c_{2}^{K} p=\sqrt{\frac{\pi}{2}} \frac{2.47}{\mathrm{Kn}}$ \\
Klinkenberg, 1941 [9] & $1+4 c \mathrm{Kn}$ & $c=1.037$ \\
Sakhaee-Pour and Bryant, 2011 [36] & $1+\frac{64}{3 \pi} \mathrm{Kn}$ & Dust gas model \\
Beskok and Karniadakis, 1999 [37] & $(1+\alpha \mathrm{Kn})\left(1+\frac{4 \mathrm{Kn}}{1-b \mathrm{Kn}}\right)$ & $\alpha=\alpha_{0} \frac{2}{\pi} \tan ^{-1} \alpha_{1} \mathrm{Kn}^{\alpha_{2}}$ \\
Civan, 2010 [10] & $(1+\alpha \mathrm{Kn})\left(1+\frac{4 \mathrm{Kn}}{1-b \mathrm{Kn}}\right)$ & $\frac{\alpha_{0}}{\alpha}-1=\frac{A}{\mathrm{Kn}^{B}}$ \\
Mohammadmoradi and Kantzas, 2016 [23] & $1+a \mathrm{Kn}$ & $a=9.62$ \\
Zhao et al., 2016 [8] & $1+4 c \mathrm{Kn}$ & $c=0.8$ \\
Florence et al., 2007 [38], Civan, 2010 [10] & $1+\frac{4 \mathrm{Kn}}{1+\mathrm{Kn}}$ & Analytical model for slip-flow regime \\
\hline \hline
\end{tabular}




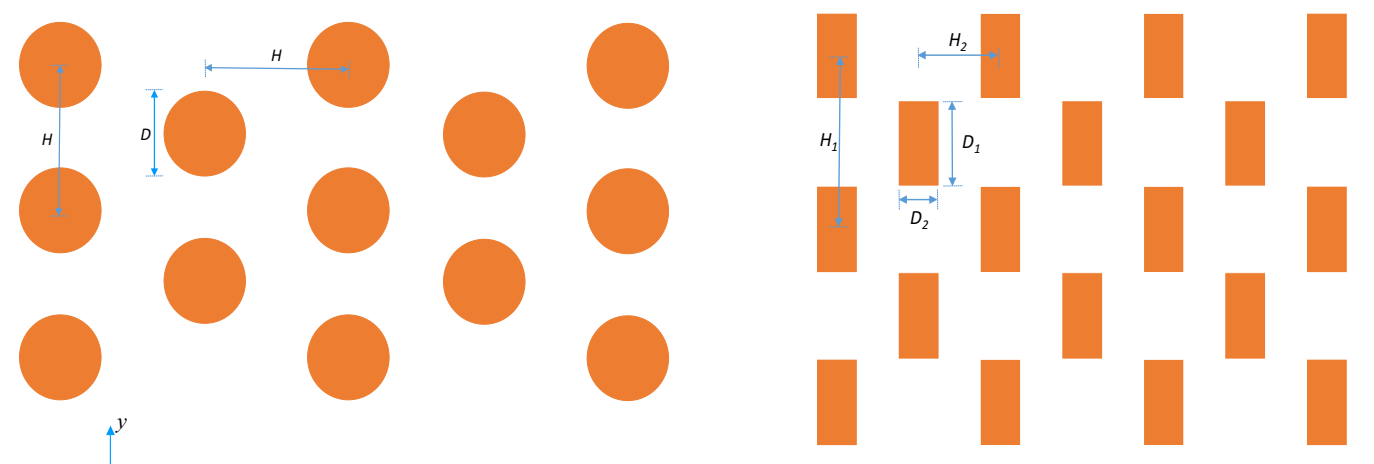

(a)

(b)

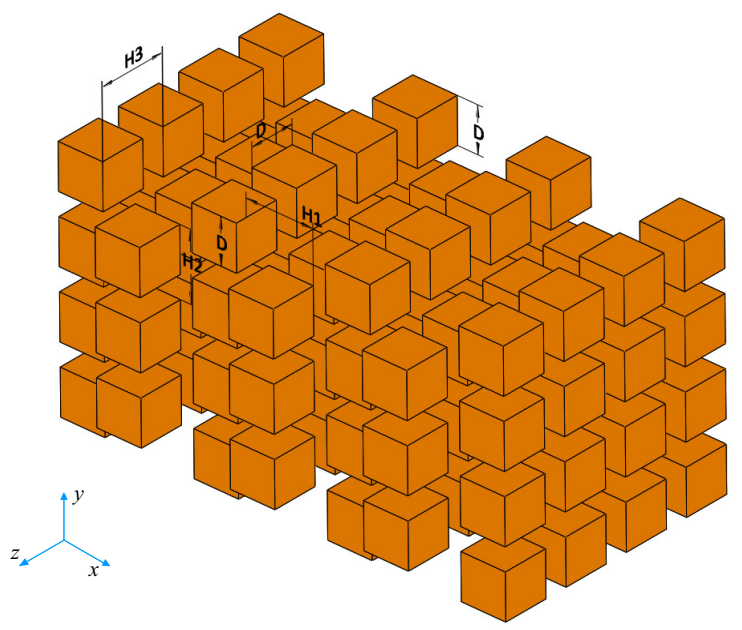

(c)

FIG. 8. Porous structures consisting of two-dimensional arranged (a) circular and (b) rectangular elements, and (c) three-dimensional arranged cubic elements.

Knudsen diffusivity coefficient from Bravo [40], and the present $D_{p}$, the predicted results by Kalarakis et al. [41] are also presented in Fig. 7 and compared with the present DSMC data at $\varepsilon=0.75$. The relative deviations of the predicted $K_{a} / K_{\infty}$ are about $9.34 \%$ and $17.9 \%$ for $\overline{\mathrm{Kn}_{p}}=2.17$ and $\overline{\mathrm{Kn}_{p}}=0.04$, respectively.

\section{Effect of pore structures}

To further check if the defined $\mathrm{Kn}_{p}$ can fully describe the Klinkenberg effect in a porous medium, simulations are performed for the periodic porous structures consisting of elements of other shapes (circle and rectangle), and also for different arrangements (both two- and three-dimensional structures). The porous structures are schematically shown in Fig. 8. The element size and the distance between neighboring elements are also varied to get different porosities and Knudsen numbers. The detailed parameters (element shape, element size, distance of neighboring elements, porosity, constriction factor, tortuosity, intrinsic permeability, and effective pore size) of the porous structures in consideration are provided in Table II. The porosity of these structures varies from 0.36 to 0.84 , the constriction factor varies from 1.060 to 1.512 , and the tortuosity varies from 0.556 to 0.926 for the different cases. The variation of $K_{a} / K_{\infty}$ with $\mathrm{Kn}_{p}$, for these different pore structures are shown in Fig. 9, where the results are found to fit well with those predicted values by Knudsen [35] with 
TABLE II. Structure parameters for the porous medium.

\begin{tabular}{|c|c|c|c|c|c|c|c|}
\hline $\begin{array}{l}\text { Element } \\
\text { shape }\end{array}$ & $\begin{array}{l}\text { Element } \\
\text { size }\end{array}$ & $\begin{array}{l}\text { Distance of } \\
\text { neighboring } \\
\text { elements }(m)\end{array}$ & Porosity & $\begin{array}{l}\text { Constriction } \\
\text { factor }\end{array}$ & Tortuosity & $\begin{array}{c}\text { Intrinsic } \\
\text { permeability } \\
\left(\mathrm{m}^{2}\right)\end{array}$ & $\begin{array}{l}\text { Effective pore } \\
\text { length }(\mathrm{m})\end{array}$ \\
\hline \multirow[t]{4}{*}{ Square } & \multirow[t]{4}{*}{$D^{\mathrm{a}} \times D$} & $2.5 \mathrm{D}$ & 0.840 & 1.060 & 0.833 & $1.268 \times 10^{-1} D^{2}$ & $1.360 D$ \\
\hline & & $2 D$ & 0.750 & 1.130 & 0.800 & $4.252 \times 10^{-2} D^{2}$ & $0.893 D$ \\
\hline & & $1.5 \mathrm{D}$ & 0.556 & 1.300 & 0.750 & $6.392 \times 10^{-3} D^{2}$ & $0.461 D$ \\
\hline & & $1.25 D$ & 0.360 & 1.510 & 0.714 & $9.168 \times 10^{-4} D^{2}$ & $0.246 D$ \\
\hline \multirow[t]{2}{*}{ Circle } & \multirow[t]{2}{*}{ Diameter $D$} & $2 D$ & 0.804 & 1.078 & 0.926 & $7.109 \times 10^{-2} D^{2}$ & $0.943 D$ \\
\hline & & $1.333 D$ & 0.558 & 1.293 & 0.894 & $5.150 \times 10^{-3} D^{2}$ & $0.345 D$ \\
\hline \multirow[t]{2}{*}{ Rectangle } & \multirow[t]{2}{*}{$D_{1}^{\mathrm{a}} \times D_{2}^{\mathrm{a}}$} & $2 D_{1} ; 2 D_{2}$ & 0.750 & 1.125 & 0.667 & $1.472 \times 10^{-2} D_{1}^{2}$ & $0.630 D_{1}$ \\
\hline & & $1.25 D_{1} ; 1.25 D_{2}$ & 0.360 & 1.512 & 0.556 & $2.561 \times 10^{-4} D_{1}^{2}$ & $0.167 D_{1}$ \\
\hline \multirow[t]{2}{*}{ Cube } & \multirow[t]{2}{*}{$D \times D \times D$} & $1.5 \mathrm{D}$ & 0.704 & 1.079 & 0.889 & $1.562 \times 10^{-2} D^{2}$ & $0.492 D$ \\
\hline & & $1.2 \mathrm{D}$ & 0.421 & 1.212 & 0.842 & $9.539 \times 10^{-2} D^{2}$ & $0.176 D$ \\
\hline
\end{tabular}

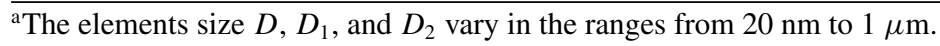

an average difference of less than 6\%. Thus, it further proves that the Klinkenberg effect in a porous medium can be fully described by using the Knudsen number characterized by the effective pore size as defined in this study.

Based on the first- and second-order Klinkenberg correlations:

$$
\begin{gathered}
K_{a} / K_{\infty}=1+a \mathrm{Kn}_{p}, \\
K_{a} / K_{\infty}=1+b \mathrm{Kn}_{p}+c \mathrm{Kn}_{p}{ }^{2},
\end{gathered}
$$

the constants of the correlations can also be correlated by the present DSMC results. Here, for Eq. (20), the correlated value of $a$ is 7.23 and the $R$-square of the correlation is 0.995. For Eq. (21),

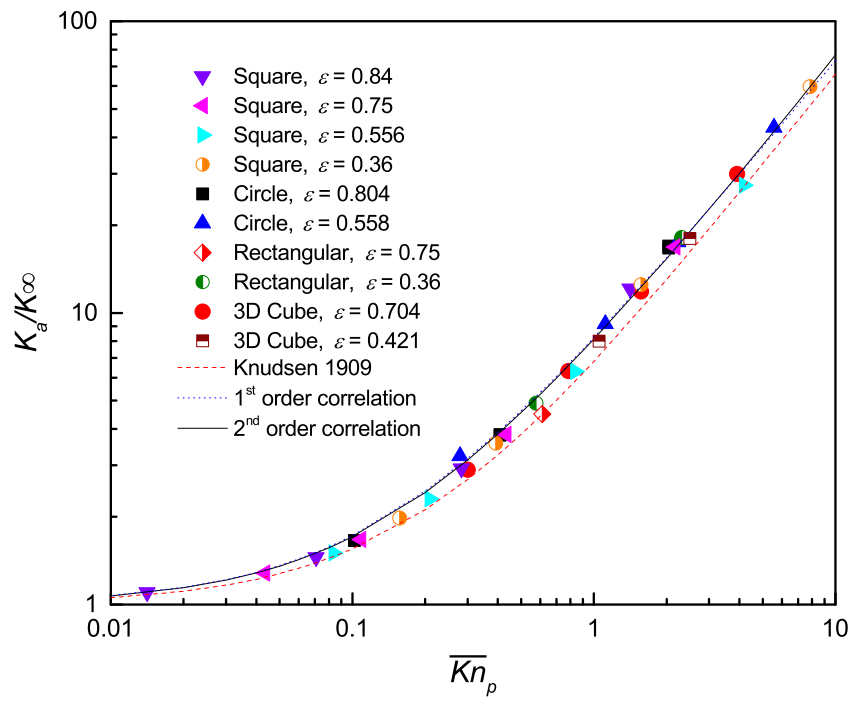

FIG. 9. Variation of $K_{a} / K_{\infty}$ with $\mathrm{Kn}_{p}$ for various pore structures [35]: first-order correlation [Eq. (20)], second-order correlation [Eq. (21)]. 
$b$ is 7.08 and $c$ is $4.85 \times 10^{-2}$, and the $R$-square of the correlation is 0.996 . The results of Eqs. (20) and (21) are also presented in Fig. 9. It can be seen the second-order correlation does not add much to the accuracy compared to the first order one in the ranges of parameters studied in this study.

\section{CONCLUSION}

Direct simulation Monte Carlo (DSMC) method has been used in this study to investigate the pressure driven gas transport characteristics through a porous medium with micro- and nanopores for the porosities of $0.36 \leqslant \varepsilon \leqslant 0.84$ and for different Knudsen numbers, based on the effective pore size, of $0.01 \leqslant \mathrm{Kn}_{p} \leqslant 10$. The effective pore size is defined as a function of the porosity, the tortuosity, the contraction factor, and the intrinsic permeability of the porous medium, which can be calculated if the structure of the porous medium is determined.

The pore scale flow patterns in the porous medium have been obtained, and the gas slip phenomena have been found at all solid walls. Both the velocities at the wall and in the pores increase along the flow direction, as the gas pressure decreases from inlet to outlet of the porous medium. For a constant element size and a constant pressure difference, the bulk velocity in the porous medium decreases with the decreasing porosity.

The apparent permeability of gas transportation is calculated based on the DSMC results and Darcy's equation. The ratio of the apparent permeability to the intrinsic permeability, $K_{a} / K_{\infty}$, for various porosities, element sizes, and porous structures are found to collapse to one curve if the Knudsen number is characterized by the effective pore size. The variation trends of $K_{a} / K_{\infty}$ with $\mathrm{Kn}_{p}$ by the available Klinkenberg correlations are similar with those of the present data, and some predicted values (e.g., Knudsen [32], Sakhaee and Bryant [33], Civian [10], Beskok and Karniadakis [34]) even show deviations of less than $10 \%$ compared to the present data. This, for the first time, has proven that the available correlations for apparent permeability, even though most of them have been derived from simple pipe or channel flows, are still applicative for flows in more complex geometries driven by large pressure differences, by using the effective pore size defined in this study.

The present results have also been validated by simulating gas transport in porous media with different shapes of unit elements, accounting for both two and three-dimensional porous structures. Therefore, this work demonstrated how the apparent permeability of a porous media can be calculated by the Klinkenberg correlations through fundamental and measurable descriptors of the pore structure. Also, it should be noted that our conclusions are based on DSMC simulations in porous structures without dead-end pores and consisting of somehow regularly and periodically distributed micro elements. How the effective pore size equation [Eq. (16)] should be modified to meet all types of natural and engineering used porous media will be further studied in our future work.

\section{ACKNOWLEDGMENTS}

Guang Yang kindly acknowledges the support of the Sino-German (CSC-DAAD) Postdoc Scholarship Program, the National Postdoctoral Program for Innovative Talents (Grant No. BX201600102), and the China Postdoctoral Science Foundation (Grant No. 2016M601591). The authors thank also Prof. Rainer Helmig, Dr. Visakh Vaikuntanathan, Dr. Alexandros Terzis, Dr. Xu Chu, and M.Sc. Kilian Weishaupt from the University of Stuttgart for their useful discussions.

\section{APPENDIX A: EFFECT OF THE COMPUTATIONAL DOMAIN LENGTH}

The effect of the length of the computational domain $(L)$ is studied, using the method in Sec. III, prior to the investigation to check if $L$ is sufficiently large, that the computed permeability of the present geometry can represent that of a porous medium with the same structures, and inlet/outlet effects are neglectable. Taking the porous structure in Fig. 1 as an example, it is composed of numerous unit cells with a size $2 H \times H$ of each cell. Different lengths of the geometry, $L=2 H$, $6 H, 12 H, 16 H$, and $20 H$, are tested, corresponding to the unit numbers of $1,3,6,8$, and 10 
TABLE III. Effect of the length of the geometry on the present results.

\begin{tabular}{lcccccccc}
\hline \hline No. of units & $P_{\text {in }}(\mathrm{MPa})$ & $P_{\text {out }}(\mathrm{MPa})$ & $D(\mathrm{~m})$ & $L(\mathrm{~m})$ & $V_{x, \text { out }}(\mathrm{m} / \mathrm{s})$ & $\overline{V_{x, \text { out }}}(\mathrm{m} / \mathrm{s})$ & $K_{a} / D^{2}$ & Deviation \\
\hline 1 & & & $1.6 \times 10^{-6}$ & 229.18 & 114.59 & 0.03419 & $51.68 \%$ \\
3 & & & $4.8 \times 10^{-6}$ & 154.84 & 77.42 & 0.06929 & $2.06 \%$ \\
6 & \multirow{2}{*}{0.25} & 0.05 & $4 \times 10^{-7}$ & $9.6 \times 10^{-6}$ & 79.10 & 39.55 & 0.07079 & $0.06 \%$ \\
8 & & & $1.28 \times 10^{-5}$ & 59.33 & 29.66 & 0.07080 & $0.07 \%$ \\
10 & & & $1.6 \times 10^{-5}$ & 47.43 & 23.72 & 0.07075 & - \\
\hline \hline
\end{tabular}

in the $X$ direction. The results for $D=400 \mathrm{~nm}, \varepsilon=0.75, P_{\text {in }}=0.25 \mathrm{MPa}$ and $P_{\text {out }}=0.05 \mathrm{MPa}$ are presented in Table III. $V_{\mathrm{x} \text {, out }}$ is the averaged velocity $V_{x}$ in the fluid regions at the outlet, and $\overline{V_{\mathrm{x} \text {,out }}}$ is the macroscopic averaged $V_{x}$ at the outlet of the whole porous medium. It can be observed that the dimensionless apparent permeability has a relative difference of $2.06 \%$ for $L=6 H$, compared to that of $L=20 \mathrm{H}$. When the length of the computational domain is further increased to $L \geqslant 12 \mathrm{H}$, it is found to have a neglectable effect on the permeability $(<0.07 \%)$. Therefore, $L=12 \mathrm{H}$ (6 units in the $X$ direction) is sufficient for the investigation of the permeability and is used in the present study.

\section{APPENDIX B: EFFECTS OF THE COMPRESSIBILITY AND THE INERTIAL FORCE}

The Mach number is calculated to check the effect of the compressibility of the fluid on the present results. The inertial effects (Forchheimer term) are also evaluated by calculating the Reynolds number at the inlet. Figure 10 shows the parameter map for all the numerical cases in the present study. For each case at a fixed $\mathrm{Kn}_{p}$, the local Mach number in the flow domain varies from 0 to $\mathrm{Ma}_{\max }$, where the $\mathrm{Ma}_{\max }$ always locates at the centerline of the outlet (can be inferred from the velocity profiles in Figs. 2 and 4). It is seen from Fig. 10 that the average Mach numbers in all cases are below 0.15. For the maximum Mach number, only four sets of data in $\overline{\mathrm{Kn}_{p}}<0.1$ have $\mathrm{Ma}_{\max }$ above 0.3 (Flows are usually treated as being incompressible when $\mathrm{Ma}<0.3$ [42]). However, as it can be seen from Figs. 7 and 9 that the variation of apparent permeability with Knudsen number is not obvious for such small Knudsen numbers. Therefore, the compressibility of the flow can be considered to have a negligible effect on the conclusions of the present study. Similarly, the Reynolds numbers at the inlets for all the cases are less than 1 , except for only three cases also at $\overline{\mathrm{Kn}_{p}}<0.1$. The same

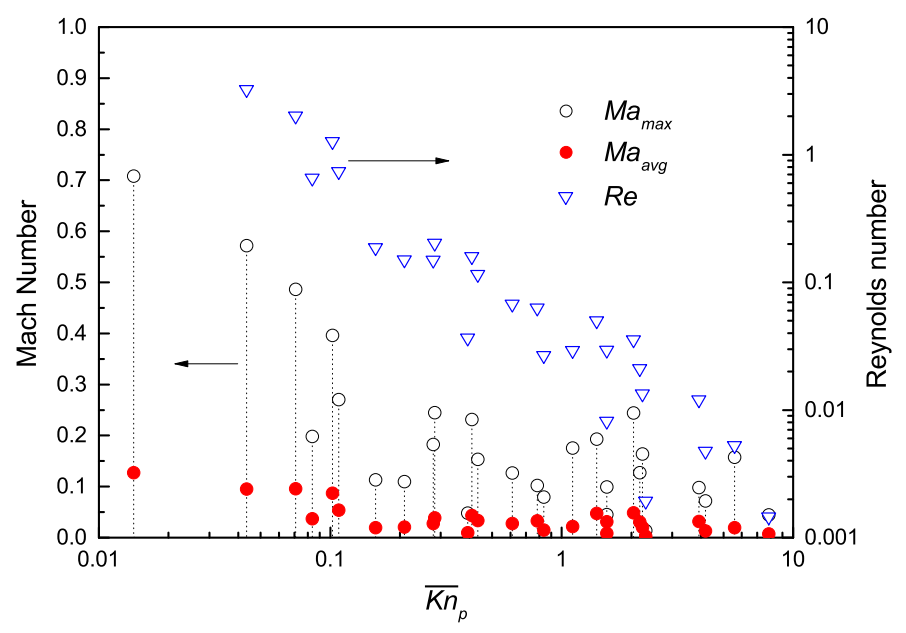

FIG. 10. Parameter map of the present study. 


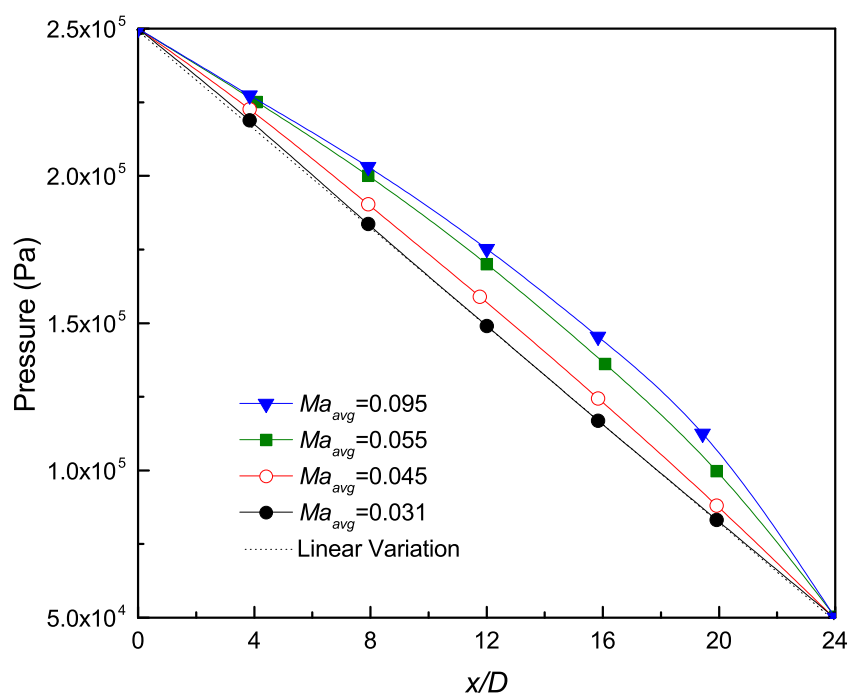

FIG. 11. Streamwise variation of the gas pressure for various Mach numbers.

conclusions can be drawn even if these data were excluded. Therefore, the inertial force also proves to have no obvious effect on the present conclusions.

The streamwise variation of cross-section averaged gas pressure from inlet to outlet is rechecked and presented in Fig. 11. As the Mach number increases (also the Reynolds number is increased), the nonlinear behavior of the pressure increases. For $\mathrm{Ma}_{\mathrm{avg}}=0.045$, the average pressure in the flow domain is $0.155 \mathrm{MPa}$, which is only $3.3 \%$ higher than $\left(P_{\text {in }}+P_{\text {out }}\right) / 2$. As can be seen from Fig. 10, only four cases in $\overline{\mathrm{Kn}_{p}}<0.1$ have average Mach numbers higher than 0.045 (those of $\left.\mathrm{Ma}_{\max }>0.3\right)$. On the other hand, even for the rare case of high Mach number of $\mathrm{Ma}_{\mathrm{avg}}=0.095$, the average pressure in the flow domain is $13 \%$ higher than $\left(P_{\text {in }}+P_{\text {out }}\right) / 2$, based on Eq. (20), that makes an error of $K_{a} / K_{\infty}$ less than $5 \%$ in the region of $\overline{\mathrm{Kn}_{p}}<0.1$. Therefore, the usage of gas properties at the pressure $\left(P_{\text {in }}+P_{\text {out }}\right) / 2$ to define the average Knudsen number in the present study can be considered reasonable.

[1] M. E. Davis, Ordered porous materials for emerging applications, Nature 417, 813 (2002).

[2] J. L. Acosta and A. F. Camacho, Porous Media: Heat and Mass Transfer, Transport, and Mechanics (Nova Science Publishers, Inc., New York, 2009).

[3] Y. C. Fang and W. W. Liou, Computations of the flow and heat transfer in microdevices using DSMC with implicit boundary conditions, J. Heat Transf. ASME 124, 338 (2002).

[4] G. A. Bird, The DSMC Method 2nd ed. (CreateSpace Independent Publishing Platform, 2013), Vol. 1.

[5] D. L. Morris, L. Hannon, and A. L. Garcia, Slip length in a dilute gas, Phys. Rev. A 46, 5279 (1992).

[6] T. J. Scanlon, E. Roohi, C. White, M. Darbandi, and J. M. Reese, An open source, parallel DSMC code for rarefied gas flows in arbitrary geometries, Comput. Fluids. 39, 2078 (2010).

[7] W. Wagner, A convergence proof for Bird's direct simulation Monte Carlo method for the Boltzmann equation, J. Stat. Phys. 66, 1011 (1992).

[8] J. Zhao, J. Yao, M. Zhang, L. Zhang, Y. Yang, H. Sun et al., Study of gas flow characteristics in tight porous media with a microscale lattice Boltzmann model, Sci. Rep. 6, 32393 (2016).

[9] L. J. Klinkenberg, The Permeability of Porous Media to Liquids and Gases, in: API-41-200 (American Petroleum Institute, New York, 1941), pp. 41-200. 
[10] F. Civan, Effective correlation of apparent gas permeability in tight porous media, Transp. Porous Media. 82, 375 (2010)

[11] E. C. Stern, I. Nompelis, T. E. Schwartzentruber, and G. V Candler, in Proceedings of the AIAA Scitech Forum, Microscale simulations of porous TPS materials: Application to permeability (AIAA Inc., Kissimmee, Florida, 2014), pp. 1-15.

[12] Y. Kawagoe, T. Oshima, K. Tomarikawa, T. Tokumasu, T. Koido, and S. Yonemura, A study on pressuredriven gas transport in porous media: From nanoscale to microscale, Microfluid. Nanofluid. 20, 162 (2016).

[13] C. Christou and S. K. Dadzie, Direct-simulation Monte Carlo investigation of a Berea porous structure, SPE J. 21, 0938 (2016).

[14] A. Borner, F. Panerai, and N. N. Mansour, High temperature permeability of fibrous materials using direct simulation Monte Carlo, Int. J. Heat Mass Transf. 106, 1318 (2017).

[15] C. White, M. K. Borg, T. J. Scanlon, and J. M. Reese, A DSMC investigation of gas flows in micro-channels with bends, Comput. Fluids. 71, 261 (2013).

[16] R. C. Palharini, C. White, T. J. Scanlon, R. E. Brown, M. K. Borg, and J. M. Reese, Benchmark numerical simulations of rarefied non-reacting gas flows using an open-source DSMC code, Comput. Fluids. 120, 140 (2015).

[17] N. Hadjiconstantinou, Analysis of discretization in the direct simulation Monte Carlo, Phys. Fluids. 12, 2634 (2000).

[18] Z. X. Sun, Z. Tang, Y. L. He, and W. Q. Tao, Proper cell dimension and number of particles per cell for DSMC, Comput. Fluids. 50, 1 (2011).

[19] M. Wang and Z. Li, Simulations for gas flows in microgeometries using the direct simulation Monte Carlo method, Int. J. Heat Fluid Flow. 25, 975 (2004).

[20] W. W. Liou and Y. C. Fang, Implicit boundary conditions for direct simulation Monte Carlo method in MEMS flow predictions, Comput. Model. Eng. Sci. 1, 118 (2000).

[21] Z. Chai, B. Shi, Z. Guo, and J. Lu, Gas flow through square arrays of circular cylinders with Klinkenberg effect: A lattice Boltzmann study, Commun. Comput. Phys. 8, 1052 (2010).

[22] Z. Wang, Y. Guo, and M. Wang, Permeability of high-Kn real gas flow in shale and production prediction by pore-scale modeling, J. Nat. Gas Sci. Eng. 28, 328 (2016).

[23] P. Mohammadmoradi and A. Kantzas, Pore-scale permeability calculation using CFD and DSMC techniques, J. Pet. Sci. Eng. 146, 515 (2016).

[24] L. Wu, M. T. Ho, L. Germanou, X. Gu, C. Liu, K. Xu et al., On the apparent permeability of porous media in rarefied gas flows, J. Fluid Mech. 822, 398 (2017).

[25] C. F. Berg, Permeability description by characteristic length, tortuosity, constriction and porosity, Transp. Porous Media 103, 381 (2014).

[26] J. Bear, Dynamics of Fluids in Porous Media (Dover Publications, New York, 1988).

[27] C. F. Berg, Re-examining Archie's law: Conductance description by tortuosity and constriction, Phys. Rev. E 86, 046314 (2012).

[28] W. Tanikawa and T. Shimamoto, Comparison of Klinkenberg-corrected gas permeability and water permeability in sedimentary rocks, Int. J. Rock Mech. Min. Sci. 46, 229 (2009).

[29] A. E. Scheidegger, The Physics of Flow Through Porous Media, 3rd ed. (University of Toronto Press, Toronto, 1974).

[30] G. Yang and J. Wu, Effect of side ratio and aiding/opposing buoyancy on the aerodynamic and heat transfer characteristics around a rectangular cylinder at low Reynolds numbers, Numer. Heat Transf. Part A Appl. 64, 1016 (2013).

[31] M. E. Aerov and O. M. Tojec, Hydraulic and Thermal Basis on the Performance of Apparatus with Stationary and Boiling Granular Layer (Himia Press, Leningrad, 1968).

[32] J. E. Drummond and M. I. Tahir, Laminar viscous flow through regular arrays of parallel solid cylinders, Int. J. Multiph. Flow. 10, 515 (1984).

[33] B. R. Gebart, Permeability of unidirectional reinforcements for RTM, J. Compos. Mater. 26, 1100 (1992).

[34] K. Yazdchi, S. Srivastava, and S. Luding, Microstructural effects on the permeability of periodic fibrous porous media, Int. J. Multiph. Flow. 37, 956 (2011). 
[35] M. Knudsen, Die Gesetze der Molekularströmung und der inneren Reibungsströmung der Gase durch Röhren, Ann. Phys. 333, 75 (1909).

[36] Sakhaee-pour and S. L. Bryant, Gas permeability of shale, in Proceedings of the SPE Annu. Tech. Conf. Exhib. (Society of Petroleum Engineers, 2011), p. 146944.

[37] G. E. K. Ali Beskok, Report: A model for flows in channels, pipes, and ducts at micro- and nanoscales, Microscale Thermophys. Eng. 3, 43 (1999).

[38] F. A. Florence, J. A. Rushing, K. E. Newsham, and T. A. Blasingame, Improved permeability prediction relations for low permeability sands, in Proceedings of the SPE Rocky Mountain Oil Gas Technology Symposium, SPE (Society of Petroleum Engineers, 2007), Vol. 18, p. 107954.

[39] S. C. Jones, A rapid accurate unsteady-state Klinkenberg permeameter, Soc. Pet. Eng. J. 12, 383 (1972).

[40] M. C. Bravo, Effect of transition from slip to free molecular flow on gas transport in porous media, J. Appl. Phys. 102, 074905 (2007).

[41] A. N. Kalarakis, V. K. Michalis, E. D. Skouras et al., Mesoscopic simulation of rarefied flow in narrow channels and porous media, Transp. Porous Media 94, 385 (2012).

[42] J. D. Anderson, Fundamentals of Aerodynamics, 4th ed. (McGraw-Hill, New York, 2007). 